\title{
Natural Image Super Resolution through Modified Adaptive Bilinear Interpolation Combined with Contra Harmonic Mean and Adaptive Median Filter
}

\author{
Suresha D \\ Research Scholar, Visvesvaraya Technological University, Belagavi, 590018, India. \\ Email: sureshasss@gmail.com \\ Prakash H N \\ Professor and Head, Department of Computer Science and Engineering, Rajeev Institute of Technology, Hassan, \\ 573201, India. \\ Email: prakash_hn@yahoo.com
}

\begin{abstract}
Super resolution is a technique to enhance the scale of image in digital image processing. The single low resolution and multiple low resolution techniques have been used by many researchers in reconstructing high resolution image. The above resolution increasing techniques are researched under spatial and frequency domain. When increased in the resolution of image, it is very important to retain the quality of image, which is the challenging task in the domain of digital image processing. Here in this paper, the super resolution architecture for single low resolution technique has been proposed to reconstruct the high resolution image by combining interpolation and restoration methods in spatial domain. The modified adaptive bilinear interpolation is proposed for interpolation and contra harmonic mean \& adaptive median filter are used for restoration of single low resolution image. The experimentation is done on standard data set show that, the results obtained from modified adaptive bilinear interpolation are competitively improved when compare to other existing single low resolution techniques in interpolation domain.
\end{abstract}

Index Terms-Super resolution, nearest neighbor, bilinear, bicubic, modified adaptive bilinear interpolation, contra harmonic mean, adaptive median filter.

\section{INTRODUCTION}

The super resolution (SR) is a promising digital image processing technique to enhance the resolution of an input image. The single low resolution (SLR) and multiple low resolution (MLR) techniques have been proposed to enhance resolution of input image. The high resolution (HR) image is reconstructed either from SLR [4-6][8-10][12-14][16-21][23-30] or MLR [7][10][26] of same scene with exposed from different angles. These techniques are explored in both spatial [2-4][9] and frequency [8] domain.

In this paper, we propose SR algorithm for SLR input image to reconstruct HR image. The modified adaptive bilinear interpolation (MABI), contra harmonic mean (CHM) and adaptive median filter (AMF) are used for interpolation and restoration of an SLR image. Initially original HR image is translated into low resolution (LR) image by using proposed interpolation technique MABI and finally the HR image is reconstructed back from SLR image by using proposed MABI, CHM \& AMF techniques. Here original HR color image serves a reference image to compare the quality of approximated HR color image. The SR algorithms for SLR input image is explored through interpolation based, learning/training based and reconstruction/restoration based algorithms in spatial domain. The interpolation based methods are primarily classified under linear, nonlinear and variation methods, where in most familiar linear methods for interpolation [1-4][9][12] are nearest neighbor, bilinear, bicubic and the recent approach is to combine fuzzy logic to traditional interpolation methods. The learning/ training [2][6][8][16][18-20][23][27][30] based SR is primarily focused on either building or using the prior dictionary/ knowledgebase of LR to HR mappings. Then use that dictionary/ knowledgebase to regenerate HR image. The reconstruction/ restoration based SR will primarily target on applying edge preservative [17][25][27][29], back projection [4][10][22][29] algorithms in developing HR image. Many researchers used interpolation algorithms for generating HR image, but the quality of interpolated image is decreased by using these methods. The goal of SR algorithm is in generating the HR and good visual quality image, which is predicted from a SLR input image. The effectiveness of SR algorithm depends on preciseness of the algorithm, i.e. the algorithm may decrease the image quality instead of increasing. So this paper proposes a SR algorithm which 
is the hybrid version of image interpolation combined with restoration.

The remaining sections of this paper is as follows: section 2 includes the related works on enhancing resolution and restoration of an image. The section 3 explains proposed architecture with protocol phases (Interpolation and Restoration) and algorithm. The results have been discussed in the section 4 with root mean squared error (RMSE), mean squared error (MSE) and peak signal to noise ratio (PSNR) computation result statistics and finally we have summarized and concluded the paper in the section 5 .

\section{RELATED WORK}

Major research work on SR is primarily focused on building dictionary/ knowledgebase in learning and training based SR. The [2] used learning based SR which uses adaptable $\mathrm{k}$ neighbours for MMSE image interpolation, where in [8] used a SLR input image and a constructed knowledgebase of LR to HR pieces using discrete wavelet transform(DWT) with inhomogeneous gaussian markov random field prior. In image hallucination [16] primal sketch priors were constructed for enhancing quality of image, a contour smoothness and reconstructive constraint is applied for construction of HR image. In patch based SR, the input LR image is divided into $3 \mathrm{X} 3$ patches [18] then interpolated by bicubic interpolation algorithm and matched with dictionary of patches, which hold HR image patch details for interpolated LR patch. In kernel based regression [19], the pairs of input LR to output HR example pair of input and output image were trained, training piece pair are arbitrarily selected for regression from a set of LR and corresponding desired HR image.

The sparse representation [20] for SR is based on piece wise construction, where each piece of LR input is constructed and the coefficients are used to produce HR image output by together training two databases one for LR image and other for HR image pieces. In nonnegative neighbour embedding [21] a dictionary of LR and HR trained piece group are used to understand the unknown HR details where LR feature vector in the input image is weighted group of $\mathrm{k}$ nearest neighbours in knowledgebase and respective $\mathrm{HR}$ feature vector is reconstructed. In the stastical learning [23] of SR problem with support vector machine given $\mathrm{N} X \mathrm{~N}$ piece from LR work is to predict 4 pixels of HR image corresponding to one center pixel. Edge focused patch based hallucination [27] is an energy function framework for finding the pieces for training set/ dictionary. When a part of input is HR in LR image [28] then SR can be constructed based on gerchberg papoulis with dynamic properties.

Most of the research of SR is on building training database, some algorithms explore on reconstruction methods for construction of HR image. In developing HR image in spatial and wavelet domain [4] the input image is interpolated using bicubic then subjected to gaussian and back projection. The back projection is one of the most striking approach for generating HR image. The adaptive gradient magnitude self-interpolation [9] uses HR gradient calculation followed by reconstruction to generate $\mathrm{HR}$ image. As the iterative back projection produces jaggy effects [17], bilateral filtering with mean shift segmentation with complex shock filtering is used for generating HR image.

The recent SR technology uses wavelet transformations. The several wavelet transformation techniques has been applied to image processing to address interpolation for better edge enhancement to avoid blocking and jazzy artifacts. The interpolation based direction adaptive discrete wavelet transform (DWT) technology is used in image compression to reduce bit and scanning redundancy [31] for texture image which reduce computation cost for shrinking and reducing blocking artifacts with better effect along the edges. One of the major variation in DWT technology is the complex wavelet transform(CWT). The CWT technology uses two filter banks namely real and imaginary filter bank, so it is termed as dual tree complex wavelet transform. The CWT provides better directional selectivity compared to DWT as it decompose input image into eight sub bands out of which two are low frequency bands and six are high frequency bands. CWT with bicubic interpolation is used in image resolution enhancement of satellite images [32] and quick bird image data [33]. Other major variation in DWT technology is the stationary wavelet transform(SWT). The DWT mechanism will decompose the inputted image into four subcomponents with their size half of the input image causing loosing details along edges. The SWT will address this issue by having subcomponents with the same size as input image. The [34] is a combination of DWT and SWT technology as SWT components are fused with DWT components for edge enhancement. The [35] is a hybrid algorithm based on DWT technology and spatial domain gaussian filtering and back propagation for image resolution enhancement.

In HR image building the input LR image is to be interpolated and for that most familiar are the variations of bicubic interpolation mechanism, which uses 16 nearby pixels for generating new values for interpolated pixel. When we use bicubic the value of interpolated pixel is dependent on 16 surrounding pixel so it introduce error between actual original image and the interpolated image along edges. To reduce error we are proposing a new approach of interpolation termed MABI which uses only seven surrounding pixels. By applying MABI error between actual original image and the interpolated image are reduced and the interpolated image is then subjected to restoration for HR image reconstruction.

\section{PROPOSED ARCHITECTURE}

The SLR and MLR techniques have been used by many researchers in reconstructing HR image. When increases in resolution of image, it is very important to retain the quality of image at the other end is a critical task in the domain of digital image processing. So, we propose the SLR technique to reconstruct the HR image 
by combining interpolation and restoration method in spatial domain. The MABI, CHM \& AMF are used for interpolation and restoration of an image. The detailed flow of SR mechanism using interpolation phase and restoration phase is shown in figure 1 .

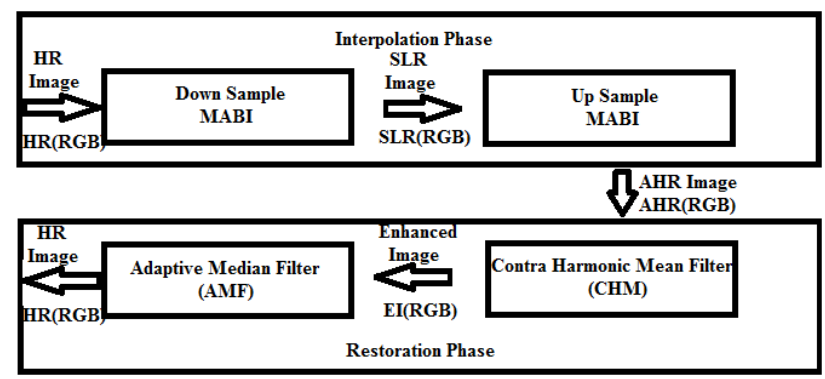

Fig.1. Proposed SR Architecture

The proposed SR architecture as shown in figure 1 accepts HR Image as input, which is also used as a reference for comparing the quality of approximated HR (AHR) image. SLR image is generated by down sampling HR image using proposed MABI interpolation function shown in figure 3 as per architecture shown in figure 4.

The SLR image is up sampled by using proposed MABI function for interpolation phase to generate AHR image. The results of MABI (AHR image with original HR) are compared with state of art interpolation algorithms nearest neighbor, bilinear and bicubic algorithms. AHR image will undergo restoration phase using $\mathrm{CHM}$ and AMF to regenerate HR image. The obtained results reveals a significant decreases in MSE and increases in PSNR using improved way of interpolation method MABI and the image quality for visual perception is enhanced through $\mathrm{CHM} \& \mathrm{AMF}$ techniques.

\section{A. Notation}

Table 1 briefs the notations used in this article for proposed SR architecture.

Table 1.Notations used in proposed architecture

\begin{tabular}{|l|l|}
\hline Notation & Explanation \\
\hline$S L R(R G B)$ & $\begin{array}{l}\text { Single low resolution colour (Red, Green, Blue) } \\
\text { image. }\end{array}$ \\
\hline$I P(R G B)$ & Input colour image. \\
\hline$O P(R G B)$ & Output colour image. \\
\hline$A H R(R G B)$ & Approximated high resolution colour image. \\
\hline$E I(R G B)$ & Enhanced high resolution colour image. \\
\hline$H R(R G B)$ & High resolution colour image. \\
\hline
\end{tabular}

\section{B. Interpolation Phase}

Figure 2 depicts the protocol used in interpolation phase for the proposed architecture shown in figure 1 .

The interpolation phase starts from accepting SLR(RGB) image from the user. The SLR(RGB) is interpolated by a factor of $\partial$ using proposed MABI function and $\mathrm{AHR}(\mathrm{RGB})$ is generated. The result of interpolation phase is subjected to restoration phase shown in figure 5.

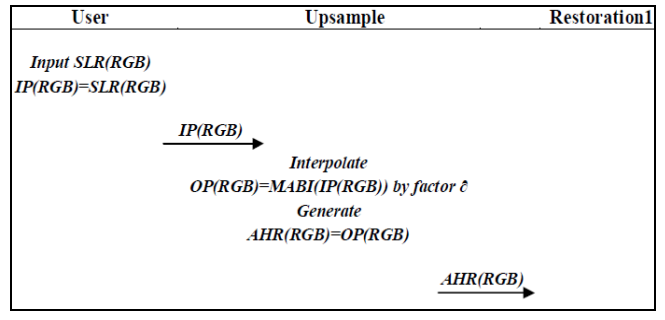

Fig.2. Protocol used in Interpolation phase

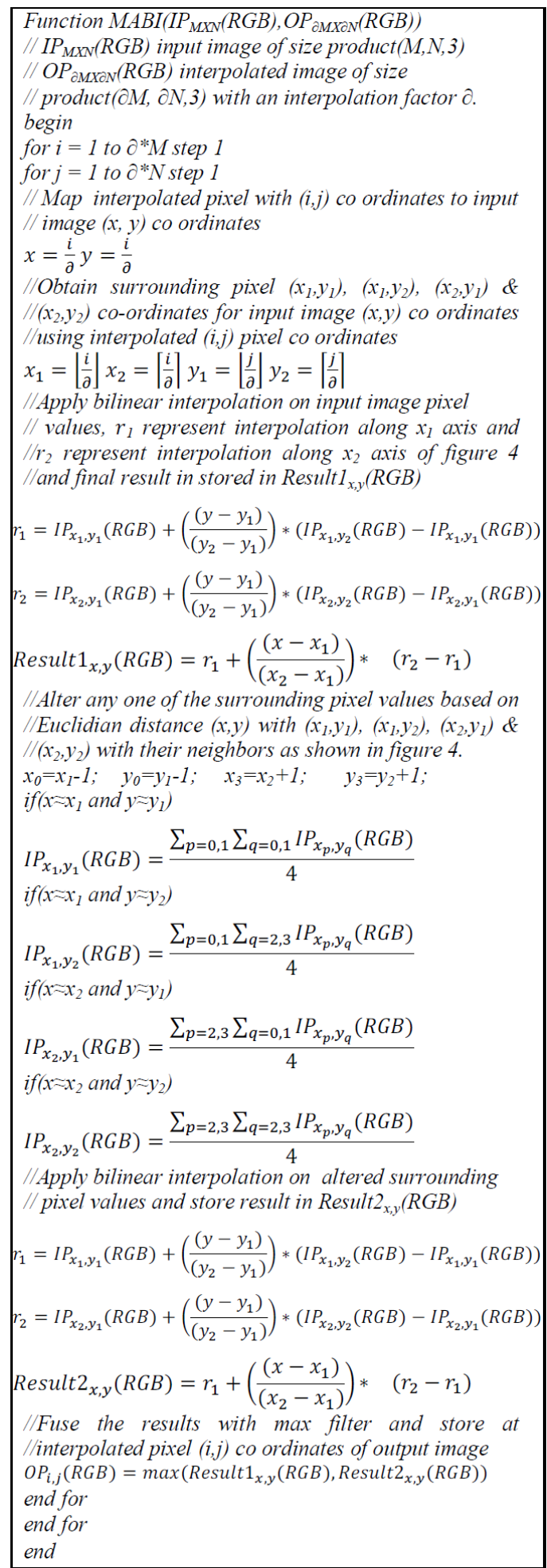

Fig.3. Function MABI 
The image interpolation function ranging from nearest neighbor to bicubic suffers from blurring edges and loosing image details, So in order to improve interpolation mechanism MABI is proposed. The figure 3 shows the implementation details for MABI architecture shown in figure 4 , which not only interpolates the input image but also increases smoothness using smoothing kernel.

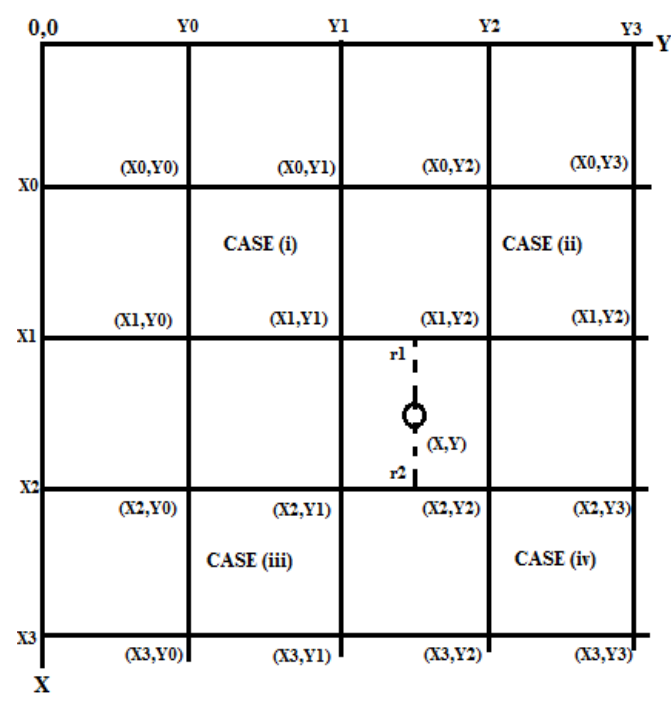

Fig.4. MABI Architecture

\section{Restoration Phase}

Figure 5 shows the protocol used in restoration phase for the proposed architecture shown in figure 1. The restoration phase is done in two levels restoration 1 and restoration 2. Restoration 1 accepts AHR(RGB) generated from interpolation phase shown in figure 2 and uses CHM filter, which reduces jaggy effect in generated AHR(RGB) using a smoothing kernel of order Q. The result of restoration 1 is $\mathrm{EI}(\mathrm{RGB})$ is feed into restoration 2 level. The restoration 2 uses AMF to generate HR(RGB). AMF is a local filter works based on non linear median filtering, which produces better results [1] than other linear filtering mechanisms. The generated HR(RGB) is visually pleasant HR image generated from SLR image using SR mechanism.

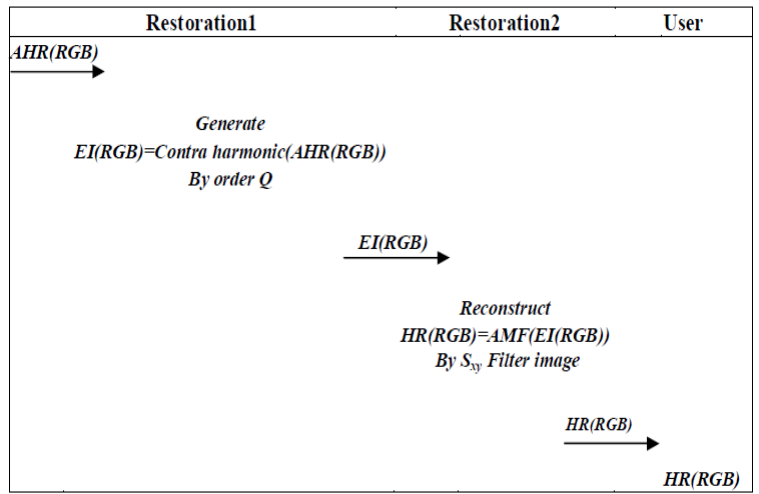

Fig.5. Protocol used in restoration phase
The $\mathrm{AHR}(\mathrm{RGB})$ image of MABI is subjected to $\mathrm{CHM}$ kernel [1][4] as shown in equation (1), which is used as smoothing kernel in spatial domain.

$$
E I(R G B)=\frac{\sum_{(s t) \in s_{\text {YY }}} A H R(R G B)(s t)^{Q+1}}{\sum_{(s t) \in s_{\text {YY }}} A H R(R G B)(s t)^{Q}}
$$

Where

AHR(RGB)-> MABI interpolated RGB image.

$\mathrm{Q}->$ Order of the filter.

EI(RGB)->Enhanced RGB image obtained after contra harmonic mean filtering.

The EI(RGB) image is subjected to AMF [1] algorithm shown in figure 6. AMF is a non linear filter to significantly reduce irregularities in generated image and sharpen the EI(RGB) image which is termed as SR image.

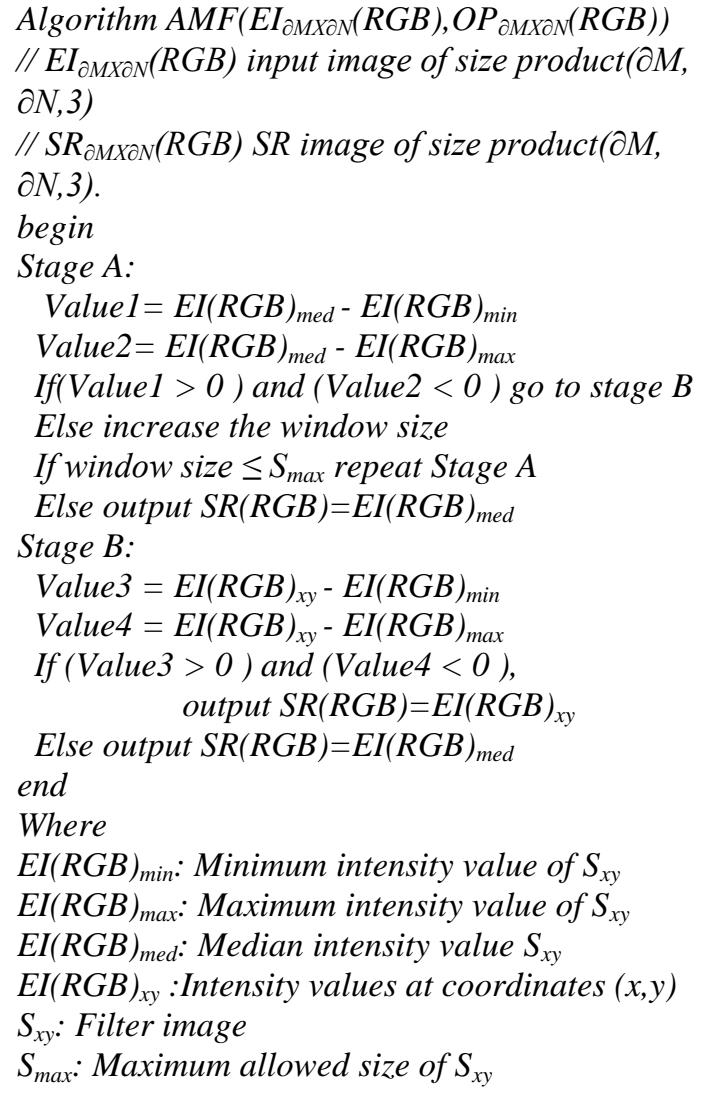

Fig.6. Algorithm for adaptive median filter

\section{RESUlTS AND DISCUSSION}

The first part of our SR algorithm involves interpolation. The set of input images shown in figure 7 are considered for experimentation on proposed MABI, nearest neighbor, bilinear and bicubic interpolation in MATLAB. The input images are referred from standard MATLAB data set. 


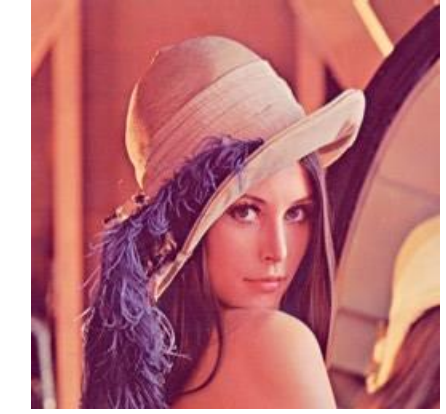

(i) Lena Size:256X256X3

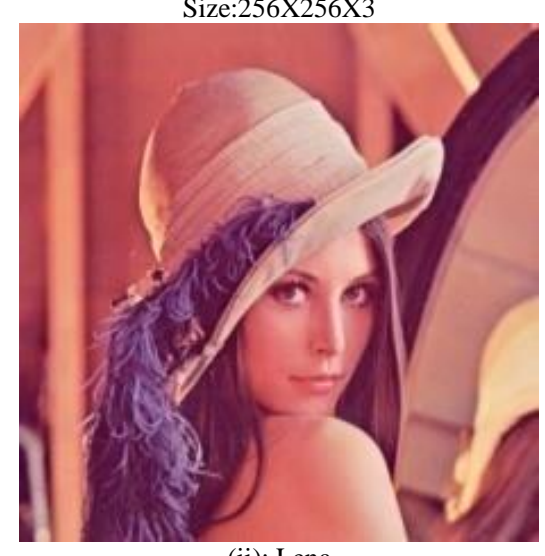

(ii): Lena

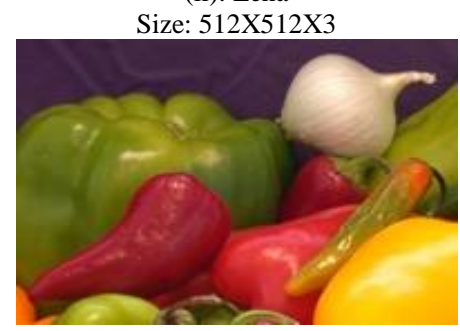

(iii): onion.png

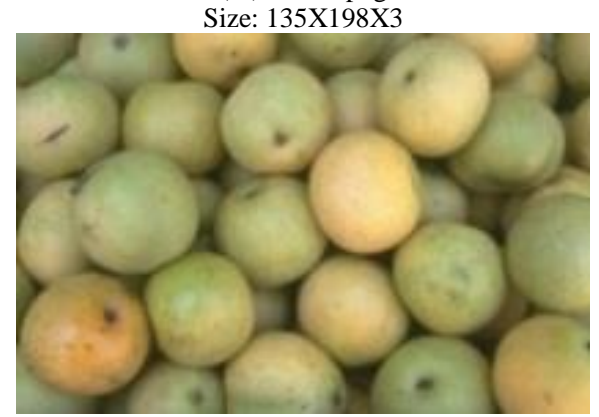

(iv): pears.png

Size: $486 \times 732 \times 3$

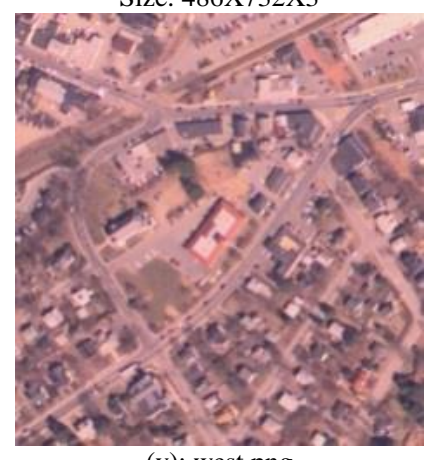

(v): west.png

Size: 394X369X3

Fig.7. Set of input images
The obtained results for figure 7 case (i) input image are shown in figure 8 and are compared with original image using RMSE, MSE and PSNR and tabulated in table 2. There is drastic decrease in RMSE, MSE and significant increase in PSNR. Which shows that our method of interpolation MABI calculate near to original image better than other techniques and competent to result of learning/training based SR algorithms.

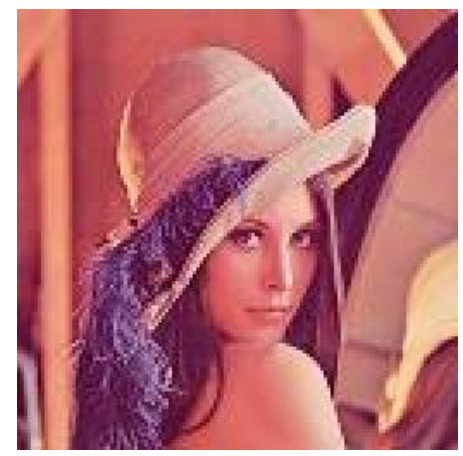

(a) Nearest neighbor

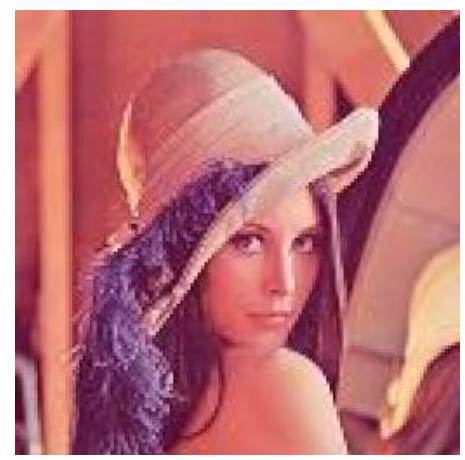

(b)Bilinear

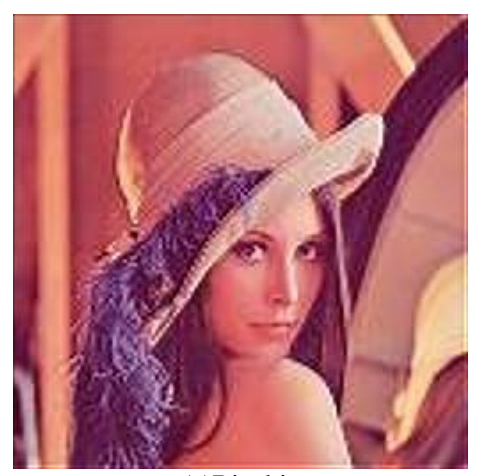

(c)Bicubic

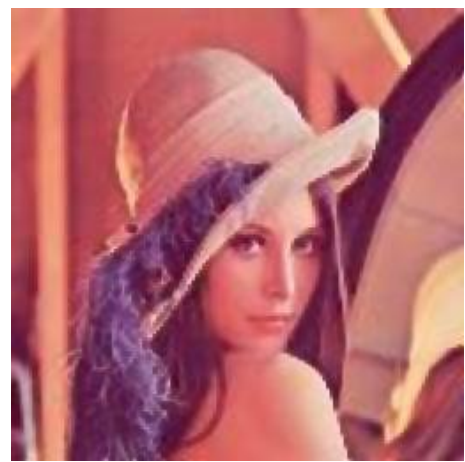

(d) MABI

Fig.8. Interpolation of input image(i) from figure 7. 
When same experiment was repeated by first down sampling by nearest neighbour, bilinear, bicubic and MABI approach by 0.5 for all input images of figure 7 and then up sampling by nearest neighbour, bilinear, bicubic and MABI approach by 2.0 respectively. The result statistics for each input image is compared with interpolated image and obtained RMSE, MSE \& PSNR values for each is shown in table 2 and performance analysed through figure 9,10 and 11 respectively.

Table 2. RMSE, MSE and PSNR Computation statistics

\begin{tabular}{|c|c|c|c|c|}
\hline \multirow[b]{2}{*}{ Image } & \multicolumn{4}{|c|}{ RMSE Tabulation } \\
\hline & $\begin{array}{c}\text { Nearest } \\
\text { Neighbor }\end{array}$ & Bilinear & Bicubic & MABI \\
\hline $\begin{array}{c}\text { Lena } \\
256 \times 256 \times 3\end{array}$ & 5.2128 & 4.8560 & 4.4997 & 3.5684 \\
\hline $\begin{array}{c}\text { Lena } \\
512 \times 512 \times 3\end{array}$ & 4.9409 & 4.3062 & 4.1357 & 3.3934 \\
\hline $\begin{array}{c}\text { onion.png } \\
\text { 135X198X3 }\end{array}$ & 4.9053 & 4.5358 & 4.2778 & 3.2784 \\
\hline $\begin{array}{c}\text { pears.png } \\
486 \times 732 \times 3\end{array}$ & 3.7416 & 2.9790 & 2.8964 & 2.2744 \\
\hline $\begin{array}{c}\text { west.png } \\
\text { 394X369X3 }\end{array}$ & 6.4076 & 6.0621 & 5.4526 & 4.4604 \\
\hline \multirow[b]{2}{*}{ Image } & \multicolumn{4}{|c|}{ MSE Tabulation } \\
\hline & $\begin{array}{c}\text { Nearest } \\
\text { Neighbor }\end{array}$ & Bilinear & Bicubic & MABI \\
\hline $\begin{array}{c}\text { Lena } \\
256 \times 256 \times 3\end{array}$ & 27.1740 & 23.5810 & 20.2475 & 12.7337 \\
\hline $\begin{array}{c}\text { Lena } \\
512 X 512 X 3\end{array}$ & 24.4127 & 18.5439 & 17.1043 & 11.5153 \\
\hline $\begin{array}{c}\text { onion.png } \\
\text { 135X198X3 }\end{array}$ & 24.0626 & 20.5733 & 18.2995 & 10.7482 \\
\hline $\begin{array}{c}\text { pears.png } \\
486 \times 732 X 3 \\
\end{array}$ & 13.9997 & 8.8744 & 8.3893 & 5.1730 \\
\hline $\begin{array}{c}\text { west.png } \\
\text { 394X369X3 }\end{array}$ & 41.0571 & 36.7493 & 29.7307 & 19.8952 \\
\hline \multirow[b]{2}{*}{ Image } & \multicolumn{4}{|c|}{ PSNR Tabulation } \\
\hline & $\begin{array}{c}\text { Nearest } \\
\text { Neighbor }\end{array}$ & Bilinear & Bicubic & MABI \\
\hline $\begin{array}{c}\text { Lena } \\
256 X 256 X 3\end{array}$ & 33.7893 & 34.4052 & 35.0671 & 37.0813 \\
\hline $\begin{array}{c}\text { Lena } \\
512 X 512 X 3 \\
\end{array}$ & 34.2546 & 35.4488 & 35.7997 & 37.5180 \\
\hline $\begin{array}{c}\text { onion.png } \\
\text { 135X198X3 }\end{array}$ & 34.3174 & 34.9978 & 35.5064 & 37.8174 \\
\hline $\begin{array}{c}\text { pears.png } \\
486 \times 732 X 3\end{array}$ & 36.6696 & 38.6494 & 38.8935 & 40.9934 \\
\hline $\begin{array}{c}\text { west.png } \\
\text { 394X369X3 } \\
\end{array}$ & 31.9969 & 32.4783 & 33.3988 & 35.1433 \\
\hline
\end{tabular}

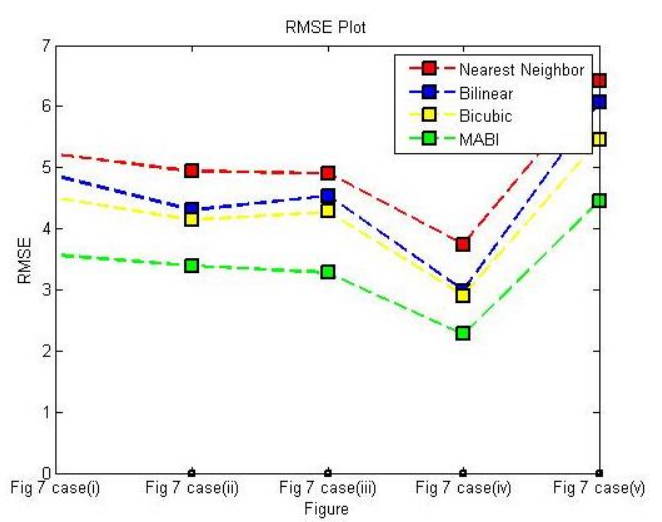

Fig.9. RMSE Performance of different interpolation methods for SR evaluated as per table 2 RMSE tabulation cost

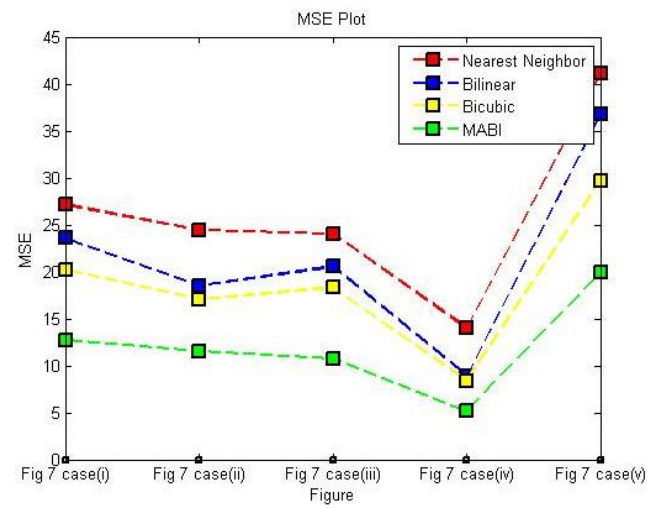

Fig.10. MSE Performance of different interpolation methods for SR evaluated as per table 2 MSE tabulation cost

The RMSE performance of figure 9 and MSE performance of figure 10 reveals designed MABI function approximates SLR to approximated HR, which very closely resembles original $H R$. The error rate between state of art algorithm is very less.

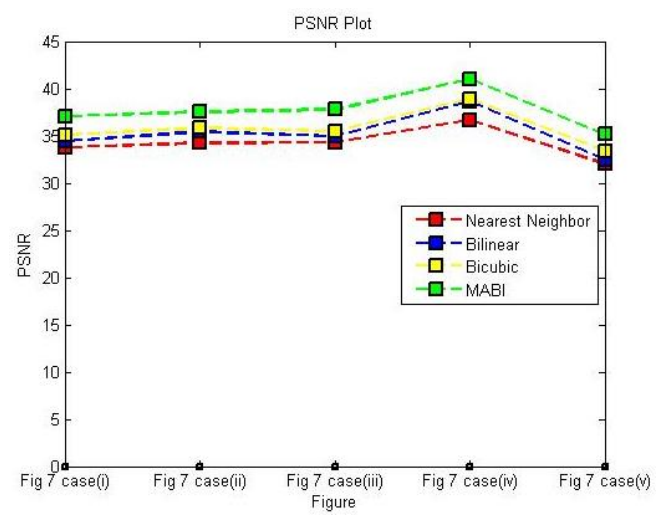

Fig.11. PSNR Performance of different interpolation methods for SR evaluated as per table 2 MSE tabulation cost measured in $\mathrm{dB}$

The analysis of figure 11 PSNR performance shows approximated signal rate is high than noise compared to other interpolation algorithms. The study of figure 9, 10 and 11 shows MABI interpolates more accurately than other interpolation techniques for super resolution in SLR interpolation domain.

The CHM kernel is applied on proposed MABI resultant image obtained in figure 8 (d) for increasing psycho visual effect. The result of CHM is sharpened using AMF to remove blurriness and introduce smoothness at edge transitions as shown in figure 12 .

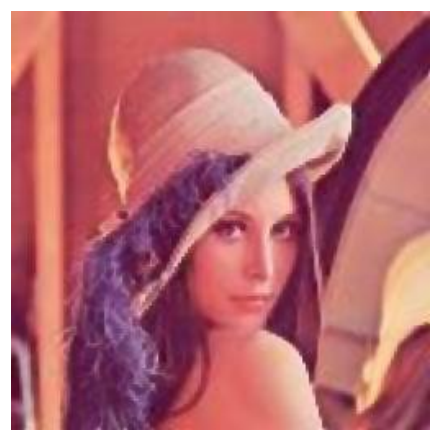

Fig.12. Reconstruction from the interpolated image 


\section{CONCLUSION}

In this paper, we have designed and implemented MABI algorithm and tested on several color images. The obtained results are compared with the current interpolation algorithms in spatial domain for single color image SR. The proposed algorithm is useful for creating HR colour image. The study of this algorithm put conclusion that interpolation algorithms in spatial domain will not exactly approximate along edges. Restoration mechanisms are needed for increasing accuracy on edges, which is done using CHM and AMF mechanisms. Competitive result statistics shows the potential of our MABI algorithm.

\section{REFERENCES}

[1] Rafael C. Gonzalez, Richard Eugene Woods, "Digital Image Processing", 3rd edition, Pearson.

[2] Karl S. Ni., Truong Q. Nguyen, "An Adaptable k-Nearest Neighbors Algorithm for MMSE Image Interpolation", IEEE Transactions on Image Processing, Vol. 18, No. 9, September 2009.

[3] Shveta Chadda, Navjeet Kaur, Rajni Thakur, "Zooming Techniques for Digital Images: A survey", IJCST, Vol 3, Issue 1, Jan-Mar2012.

[4] Sapan Naik, Nikunj Patel, " Single Image Super Resolution in Spatial and Wavelet Domain", The International Journal of Multimedia \& Its Applications (IJMA), Vol.5, No.4, August 2013.

[5] Jian Sun, Jian Sun, Zongben Xu, Heung-Yeung Shum, "Image Super-Resolution using Gradient Profile Prior". [Courtesy of http://research.microsoft.com/enus/um/people/jiansun/papers/GradientSR_CVPR08.pdf]

[6] William T. Freeman, Thouis R. Jones, and Egon C. Pasztor, "Example-Based Super-Resolution", IEEE, March/April 2002.

[7] Assaf Zomet, Alex Rav, Acha Shmuel Peleg, "Robust Super-Resolution", IEEE 2001. [Courtesy of http:// www.cs.huji.ac.il/ peleg/papers/cvpr01-RobustSR.pdf]

[8] Prakash P. Gajjar and Manjunath V. Joshi, "New Learning Based Super-Resolution: Use of DWT and IGMRF Prior", IEEE Transactions on Image Processing, Vol. 19, No. 5, May 2010, Page No: 1201-1213.

[9] Lingfeng Wang, Shiming Xiang, Gaofeng Meng, Huaiyu $\mathrm{Wu}$ and Chunhong Pan, "Edge-directed Single Image Super-resolution via Adaptive Gradient Magnitude Selfinterpolation", IEEE Transactions on Circuits and Systems for Video Technology, Vol. 23, No. 8, August 2013, Page No: 1289-1299.

[10] Amisha J. Shah, Suryakant B. Gupta, "Image Super Resolution-A Survey", 1st International Conference on Emerging Technology Trends in Electronics, Communication and Networking, 2012.

[11] Lexing Xie, "Image Restoration". [Courtesy of http://www.ee.columbia.edu/ xlx/ee4830/notes/lec7.pdf]

[12] Alexey Lukin, Andrey S. Krylov, Andrey Nasonov, "Image Interpolation by Super-Resolution". [Courtesy of https://www.researchgate.net/profile/Alexey_Lukin/public ation/228784370_Image_interpolation_by_superresolution/links/02e7e5237b71e14092000000]

[13] Andrei Rares, Marcel J. T. Reinders, Jan Biemond, "Edge-Based Image Restoration", IEEE Transactions on Image Processing, Vol. 14, No. 10, October 2005.
[14] Taeg Sang Cho, C. Lawrence Zitnick, Neel Joshi, Sing Bing Kang, Richard Szeliski, William. T. Freeman, "Image restoration by matching gradient distributions", Digital Object Identifier 10.1109/TPAMI.2011.166, Pattern Analysis and Machine Intelligence, IEEE transactions, Volume: 34, Issue: 4, April 2012, Pages: 683-694.

[15] Todd Wittman, "Mathematical Techniques for Image Interpolation". [Courtesy of http://public-digitallibrary.googlecode.com/svn/trunk/DSP/ImageResampling /Mathematical Techniques for Image Interpolation.pdf]

[16] Jian Sun, Nan-Ning Zheng, Hai Tao, Heung-Yeung Shum, "Image Hallucination with Primal Sketch Priors", Computer Vision and Pattern Recognition, 2003. Proceedings. 2003 IEEE Computer Society Conference, Volume:2, 18-20 June 2003, Pages:II-729-736 .

[17] Gaurav Hansda, "Super-Resolution with Better Edge Enhancement", University of Texas, Nov-2012.

[18] Jianchao Yang, John Wright, Yi Ma, Thomas Huang, "Image Super-Resolution as Sparse Representation of Raw Image Patches", Computer Vision and Pattern Recognition, CVPR 2008. IEEE Conference, 23-28 June 2008, Pages: 1-8.

[19] Kwang In Kim and Younghee Kwon, "Single-Image Super-resolution using Sparse Regression and Natural Image Prior", Pattern Analysis and Machine Intelligence, IEEE Transactions, Volume:32, Issue: 6 , June 2010, Pages:1127-1133.

[20] Jianchao Yang, Wright J. Huang, T.S. Yi Ma, "Image Super-Resolution Via Sparse Representation", Image Processing, IEEE Transactions, Volume: 19, Issue: 11, 2010.

[21] Marco Bevilacqua, Aline Roumy, Christine Guillemot, Marie-Line Alberi Morel, "Low-Complexity SingleImage Super-Resolution based on Nonnegative Neighbor Embedding". [Courtesy of http://people.rennes.inria.fr/ Aline.Roumy/publi/12bmvc_Bevilacqua_lowComplexity SR.pdf]

[22] Neil Alldrin, "Super-Resolution". [Courtesy of http://vision.ucsd.edu/ nalldrin/research/ superresolution/superresolution.pdf]

[23] Karl S. Ni, Sanjeev Kumar, Nuno Vasconcelos, Truong Q Nguyen, "Single Image Super resolution based on Support Vector Regression", Acoustics, Speech and Signal Processing, ICASSP 2006 Proceedings. 2006 IEEE International Conference, Volume:2, 14-19 May 2006, Pages:II.

[24] Liang-Jian Deng, Weihong Guoy, Ting-Zhu Huang, "Single Image Super-Resolution via an Iterative Reproducing Kernel Hilbert Space Method".[Courtesy of $\mathrm{ftp} / / / 128.97 .4 .253 / \mathrm{pub} / \mathrm{camreport} / \mathrm{cam} 14-26 . \mathrm{pdf}]$

[25] Qiang Zhou, Shifeng Chen, Jianzhuang Liu, Xiaoou Tang," Edge-Preserving Single Image Super-Resolution". [Courtesy of http://mmlab.ie.cuhk.edu.hk/archive/2011 $/ \mathrm{mm} 11$ _edge_preserving_SR.pdf]

[26] Lyndsey C. Pickup, "Machine Learning in Multi-frame Image Super-resolution", Michaelmas Term, 2007. [Courtesy of http://www.robots.ox.ac.uk/ vgg/ publications/2008/Pickup08/pickup08.pdf]

[27] Jian Sun, Jiejie Zhu, Marshall F. Tappen, "ContextConstrained Hallucination for Image Super-Resolution", Computer Vision and Pattern Recognition (CVPR), 2010 IEEE Conference, 13-18 June 2010, Pages: 231-238.

[28] Eran Gur, Zeev Zalevsky, "Single-Image Digital SuperResolution A Revised Gerchberg-Papoulis Algorithm", IAENG International Journal of Computer Science, 34:2, IJCS_34_2_14, 2007. 
[29] Shengyang Dai, Mei Han, Ying Wu, Yihong Gong, "Bilateral Back-Projection for Single Image Super Resolution". Multimedia and Expo, 2007 IEEE International Conference, 2-5 July 2007, Pages: 1039 1042.

[30] Oisin Mac Aodha, Neill D.F. Campbell, Arun Nair, and Gabriel J. Brostow, "Patch Based Synthesis for Single Depth Image Super-Resolution". [Courtesy of http://discovery.ucl.ac.uk/1371091/1/campbell_eccv12_de pth_super_res.pdf]

[31] Zhijun Fang, Naixue Xiong, Laurence T. Yang, Xingming Sun, and Yan Yang, "Interpolation-Based DirectionAdaptive Lifting DWT and Modified SPIHT for Image Compression in Multimedia Communications", IEEE systems journal, vol. 5, no. 4, December 2011, Page No: $584-593$.

[32] Turgay Celik and Tardi Tjahjadi, "Image Resolution Enhancement Using Dual-Tree Complex Wavelet Transform", IEEE geoscience and remote sensing letters, Vol. 7, No. 3, July 2010, Page No: 554- 557.

[33] Hasan Demirel and Gholamreza Anbarjafari, "Satellite Image Resolution Enhancement Using Complex Wavelet Transform", IEEE geoscience and remote sensing letters, Vol. 7,No.1,January 2010, Page No: 123- 126.

[34] Hasan Demirel and Gholamreza Anbarjafari, "IMAGE Resolution Enhancement by Using Discrete and Stationary Wavelet Decomposition", IEEE transactions on image processing, Vol. 20, No. 5, May 2011, Page No: $1458-1460$.

[35] Shen Chuan Tai, Tse-Ming Kuo, Chon-Hong Iao, and Tzu-Wen Liao, "A Fast Algorithm for Single-Image Super Resolution in both Wavelet and Spatial Domain", 2012 International Symposium on Computer, Consumer and Control, 2012 IEEE, DOI 10.1109/IS3C.2012.182, Page No: 699- 702.
Belagavi, India in 2001 \& 2010 respectively. He is currently a research scholar - pursuing Ph.D. from Visvesvaraya Technological University, Belagavi, India.. He has authored several peer reviewed national and international conferences \& Journal papers and his research interest includes Image processing, Computer Vision and Artificial Intelligence.

Prof. Suresha is a life member of professional bodies Computer Society of India, Indian Society for Technical Education.

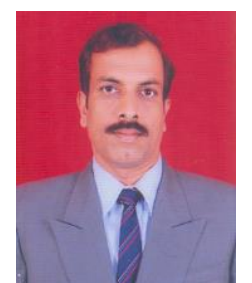

H.N. Prakash received the B.E. degree in Electronics and Communication Engineering from the University of Mysore, Mysore, India, in 1990 and the M.Tech degree in Electronic Instrumentation from Regional Engineering College, Warangal, India in 1995 respectively. He received the Ph.D. degree in Computer Science and Engineering from the University of Mysore, Mysore, India, in 2010.

He is currently a Professor in the Department of Computer Science and Engineering at Rajeev Institute of Technology, Hassan, India. He has authored several peer reviewed papers at national and international conferences and Journals Including IEEE Transactions. His research interest includes signature analysis and retrieval, clustering, biometrics, Image processing, Pattern recognition and symbolic data analysis.

Dr. Prakash is a life member of Indian professional bodies such as Institute of Engineers, Indian Society for Technical Education (ISTE) and System Society of India (SSI) and Member of Indian Institute of Engineers, India.

\section{Authors' Profiles}

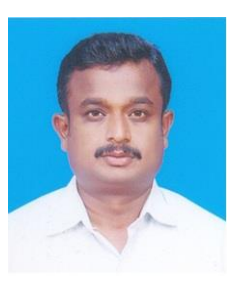

Suresha D is currently a Associate Professor in the Department of Computer Science and Engineering at Canara Engineering College, Mangalore, India. He received the B.E. \& M.Tech degree in Computer Science \& Engineering from Kuvempu University, Shankaragatta, and Visvesvaraya Technological University,

How to cite this paper: Suresha D, Prakash H N,"Natural Image Super Resolution through Modified Adaptive Bilinear Interpolation Combined with Contra Harmonic Mean and Adaptive Median Filter", International Journal of Image, Graphics and Signal Processing(IJIGSP), Vol.8, No.2, pp.1-8, 2016.DOI: 10.5815/ijigsp.2016.02.01 